\title{
Effects of silicon in plants with particular reference to horticultural crops - Review article
}

\author{
Bat-Erdene, O. ${ }^{1,2}$, Szegő, A. ${ }^{1}$, Gyöngyik, M. ${ }^{1}$, Mirmazloum, I.S. ${ }^{1}$ \& Papp, I. ${ }^{1}$ \\ ${ }^{1}$ Hungarian University of Agriculture and Life Sciences, Department of Plant Physiology and Plant Ecology, \\ 1118 Budapest, Ménesi str 44, Hungary \\ ${ }^{2}$ Mongolian University of Life Science, Plant Protection Research Institute, Forest, \\ Pastures conservation-restoration laboratory, 210153, Ulaanbaatar, Mongolia \\ Author for correspondence: oyuntogtokhb@gmail.com
}

\begin{abstract}
Summary: Silicon (Si) has long been considered as non-essential element for plant's growth and production. Numerous efforts are being made for the discovery of its beneficial effects with large scale studies laying foundation for new findings and hypotheses. Therefore, Si has been suggested to be a quasi-essential element due to its positive effects against biotic and abiotic stresses alike. Though Si is the second most abundant element in the soil profile, its availability to plants is limited to the form of monosilicic acid only. Besides, plants' ability to take-up Si and use it in their physiological processes also depends on the available transporters associated with it. Thus, the present review covers uptake and transport of silicon in plants as well as Si mediated physiological processes, including mechanisms underlying induced tolerance against biotic and abiotic stresses with a particular emphasis on horticultural species.
\end{abstract}

Bat-Erdene, O., Szegö, A., Gyöngyik, M., Mirmazloum, I.S., Papp, I. (2021): Effects of silicon in plants with particular reference to horticultural crops - Review article. International Journal of Horticultural Science 27: 95-105. https://doi.org/10.31421/ijhs/27/2021/9096

Key words: silicon, higher plants, biotic stress, abiotic stress, Si transporters

\section{Introduction}

The main constraints for agricultural production include abiotic (Meena et al., 2017) and biotic stresses (Compant et al., 2005), which limit cultivation, quality and yield of crops.

Si plays a major role in several plants' life cycle, with one of these roles to improve plant growth and yield especially in stress conditions. Some of the most important functions of $\mathrm{Si}$ include promoting plant photosynthesis by exposing leaves to light, increasing resistance to disease and pathogens, and regulating metal toxicities as well as salinity and drought stresses. Others include protection against extreme temperatures, coping with insufficient mineral composition and detrimental enzyme activities etc., (Ma, 2004; Zhu \& Gong, 2014; Adrees et al., 2015). Generally, it has been shown to have an important role in proper physiological activity, structure formation and improved plant survival in several plant species upon exposure to various biotic and abiotic stresses (Liang et al., 2015). Plant growth is dependent upon the availability of essential elements in the soil, while further minerals may be beneficial or toxic (Bienert et al., 2008). Elements categorized into the toxic group unconstructively affect plant growth, contrary to essential elements that are critical for all plants in various growth conditions. Beneficial elements are vital for some plant species in certain growth conditions. The beneficial effects of $\mathrm{Si}$ have been well documented in different plant species. Even though the essentiality of Si has always been a debate, regardless of its beneficial use and consideration by many authors as well as studies conducted on numerous plants. Nevertheless, $\mathrm{Si}$ is not only having positive effects on plants themselves but also benefits human health through food products derived from $\mathrm{Si}$ exposed plants by offering greater bone strength and improved nervous and immune system (Farooq \& Dietz, 2015).

\section{The forms of Si and its occurrence}

Silicon is a nonmetallic chemical element in the carbon family, which rarely exists in its pure form. The oxidized form of $\mathrm{Si}$ is called silicium dioxide and silicates are common in Earth's crust and particularly important component of Earth's mantle. It is found practically in all rocks, sand, clays and other soil types. Its compound also occurs in all kinds of natural waters, in the atmosphere as siliceous dust, in many plants as well as in the skeleton, tissues and body fluids of some animals.

Earth surface is covered with $27.7 \%$ of silicon, being next abundant to oxygen (46.6\%) (Mitra, 2015). Generally, 50 - 400 $\mathrm{g}$ of $\mathrm{Si}$ are contained per $\mathrm{kg}$ of soil. There are several types of $\mathrm{Si}$ forms available, including quartz and crystalline combined silicates, secondary or clay and Si-rich minerals, as well as amorphous silica that is present in most soils (Orlov, 1985). They constitute a major portion of the soil in the form of aluminum or other silicates, which account for $90 \%$ of the earth's crust by mass. Generally, all Si forms are sparingly soluble and biogeochemically inert. Principal soluble forms of silicon in soil are monosilicic and polysilicic acid (Mica, 1986).

Monosilicic acid occurs in weakly adsorbed state in the soil (Matichenkov, 1990; Matichenkov et al., 1997) and has a low 
capacity for migration down the soil profile (Khalid \& Silva, 1980). It can interact with aluminum, iron, manganese and heavy metals to form slightly soluble silicates (Horiguchi, 1988; Lumsdon \& Farmer, 1995). Unlike monosilicic acid, polysilicic acid acts as an adsorbent. It is an integral component of the soil solution and mainly affects soil physical properties and it is important for the formation of soil structure (Matychenkov et al., 1995).

According to tissue analysis studies conducted in a wide variety of plants, Si concentration ranged from 1 to $100 \mathrm{~g} \mathrm{~kg}^{-1}$ of dry weight (Farooq \& Dietz, 2015). These values of silicon present in plants are equivalent to macroelements such as phosphorous, nitrogen and calcium. Among plants, rice (Oryza sativa) $150-300 \mathrm{~kg} \mathrm{ha}^{-1}$, and wheat (Triticum spp.) $50-150 \mathrm{~kg}$ $\mathrm{ha}^{-1}$ absorbed the largest amount of Si (Datnoff et al., 2001) in the field. Moreover, dicotyledonous plants including cucumber (Cucumis sativus) (Liang et al., 2005; Nikolic et al., 2007) absorb Si actively (Liang et al., 2006). Nonetheless, some plants like tomato (Solanum lycopersicum), bean (Phaseolus vulgaris) and several others absorb $\mathrm{Si}$ passively from soil solution (Mitani \& Ma, 2005; Liang et al., 2005, 2006; Nikolic et al., 2007).

\section{Silicon uptake and accumulation in plants}

Incorporation of Si by plants depends on the uptake ability and permits potential beneficial effects (Takahashi et al., 1990; Ma, 2004). Thus, plants are classified into three groups based on their variable capacity to accumulate silicon under average field conditions. These include high accumulators $(>5 \%)$, intermediate accumulators $(1 \%)$, and low accumulators $(<0.1 \%$ of their dry weight basis), such as rice, cucumber and tomato respectively (Deshmukh \& Bélanger, 2016). Monocot plants tend to accumulate higher tissue concentrations of $\mathrm{Si}$ than dicots (up to 10\%) (Table 1).

Plant roots absorb $\mathrm{Si}$ in the form of monosilicic acid $\left(\mathrm{H}_{4} \mathrm{SiO}_{4}\right)$ at concentrations of $0.1-2.0 \mathrm{mM}$. Monosilicic acid crosses plasma membranes only at a certain $\mathrm{pH}$ range (Epstein, 1994; Raven, 2001; Ma \& Yamaji, 2006). Due to significant differences among plant species, three possible types of $\mathrm{Si}$ uptake mechanisms have been proposed by Takahashi et al. (1990). Plants having higher Si uptake levels than would be expected by water transport are classified as active, plants with similar Si and water uptake rates are classified as passive, whereas those with lower Si uptake than water are referred as rejective types. High accumulators are expected to show active Si uptake from available resources.

Remarkably, molecular studies have found a large group of genes and respective proteins in diverse species that are responsible for $\mathrm{Si}$ absorption and transport. These $\mathrm{Si}$ transporters allow $\mathrm{Si}$ translocation from soil solution into the root and further into the shoot. (Ma \& Yamaji, 2015). Si transporters are specific transmembrane proteins that mediate the uptake mechanism (Marron et al., 2016).

The very first $\mathrm{Si}$ transporter was identified in rice. This discovery suggested that a specific system for an active $\mathrm{Si}$ transport existed. Identification and cloning of the Lsil (Low silicon 1) transporter gene occurred by studying a rice mutant (Ma et al., 2004). This transporter gene localized on chromosome 2 , and its protein product belonged to the aquaporin subfamily (Gomes et al., 2009) of the NIP3 (Nod 26-like major intrinsic protein 3) major protein family. These proteins facilitate transmembrane movements of small molecules. Presumably LSI1, when present in the plasma membrane of exo- and endodermis, promotes Si uptake into the root stele. Without this transporter uptake is prevented by the presence of Casparian strip. Lsil is expressed predominantly in the main and lateral roots but not in root hairs, which is consistent with a previous study showing no participation of root hairs in the $\mathrm{Si}$ uptake process (Ma et al., 2001). Thus, LSI1 is proven to be a major influx transporter of $\mathrm{Si}$ into rice root cells.

Further studies identified LSI2 as another Si transporter, which is potentially accountable for active loading Si from the exo- and endodermis to the cortex and stele respectively in rice (Ma et al., 2007, 2011). LSI2 is located on the plasma membrane of exo- and endodermal cells at the proximal side and builds up in the mature root zone. Its localization is consistent with its role as being an efflux transporter for silicic acid. Another influx transporter LSI6, was found expressed in the parenchyma cells of the leaves. Presumably, this transporter moves $\mathrm{Si}$ across vascular bundles in shoot.

Homologous genes to these transporters of rice have also been found in other monocots and in dicot plants, such as soybean (Glycine max) (Deshmukh et al., 2013), pumpkin (Cucurbita moschata) (Mitani et al., 2011) and cucumber (Cucumis sativus) (Wang et al., 2014; Sun et al., 2017, 2018).

\section{Effects of silicon in biotic stress situations}

Biotic stress is caused by pathogens, insect pests and other living organisms. Plants have developed defense mechanisms to overcome these biotic stresses. Si increased resistance of plants against biotic stresses as has been documented by various studies. Many scientists laid a foundation for hypotheses regarding different defense mechanisms (Figure 1). The first hypothesis rooted from work of Wagner (1940), who suggested that $\mathrm{Si}$ acted as a mechanical barrier when deposited on the tissue surface, which prevented the penetration of pathogens into different host plants. Another hypothesis was that plants prevented the propagation of pathogens by accumulating phenolic like material when Si is applied (Samuels et al., 1991; Chérif et al., 1992). This hypothesis has been further analyzed and supported by Fawe et al. (1998) with the interaction of $\mathrm{Si}$ treated cucumber and powdery mildew infection.

Interestingly, field and experimental data suggest that $\mathrm{Si}$ triggered benefit in biotic stress is limited to biotrophic (e.g. powdery mildew) and hemi-biotrophic (e.g. rice blast) pathogens. In recent years the importance of effector proteins has been highlighted especially in case of biotrophic and hemibiotrophic host-pathogen interactions. According to Giraldo \& Valent (2013), effectors can modify host cell ultrastructure, metabolism and function by interfering with relevant signaling pathways. Fungal effectors are released into the apoplast and translocated into the cytoplasm through extrahaustorial matrix and the cell membrane (Bozkurt et al., 2012). This makes the apoplast a likely interaction site for effectors and plant targets (Wang \& Wang, 2018). In plants, $\mathrm{SiO}_{2}$ deposition also located in apoplast, more specifically at the interface of the plasma membrane and the cell wall (Bauer et al., 2011; Zhang et al., 2013). Number of studies support the evidence that Si interferes with effectors or receptors in order to contribute to plant resistance (Ghanmi et al., 2004; Rasoolizadeh et al., 2018). Therefore, Si fertilizations are considered as a better alternative to fungicides and supported by numerous studies for controlling powdery mildew in variety of horticultural crops such as cucumber, zucchini (Vercelli et al., 2017), melon (Dallagnol et al., 2012), strawberry (Liu et al., 2020) and tomato (Garibaldi et al., 2011). 
Table 1. Si concentration and Si transporters identified in some important crops

\begin{tabular}{|c|c|c|c|}
\hline Plant name & Species & $\begin{array}{l}\text { Mean } \mathrm{Si} \text { concentration in } \\
\text { shoots }\left(\mathrm{g} \mathrm{kg}^{-1} \mathrm{DW}\right)\end{array}$ & Si transporters described \\
\hline Rice & Oryza sativa & 41.67 & OsLsi1, OsLsi2, OsLsi3, OsLsi6 \\
\hline Horse tail & Equisetum arvense & 39.92 & EaLsi2-1, EaLsi2-2, EaNIP3;1, EaNIP3;1 \\
\hline Cucumber & Cucumis sativus & 19.66 & CsLsi1, CsLsi2, CSiT1, CSiT2 \\
\hline Sunflower & Helianthus annuus $\mathrm{L}$ & 18.76 & - \\
\hline Tomato & Solanum lycopersicum & 15.45 & - \\
\hline Soybean & Glycine $\max$ & 13.99 & GmNIP2-1, GmNIP2-2 \\
\hline Common bean & Phaseolus vulgaris & 9.49 & - \\
\hline Watermelon & Citrullus lanatus & 8.37 & - \\
\hline Maize & Zea mays subsp. & 8.27 & ZmLsi1, ZmLsi2, ZmLsi6 \\
\hline Wax gourd & Benincasa hispida $\mathrm{L}$ & 3.22 & - \\
\hline Onion & Allium сера & 0.62 & - \\
\hline Horseradish & Armoracia rusticana & 0.58 & - \\
\hline Potatoes & Solanum tuberosum & 0.4 & StLsil \\
\hline Pepper & Capsicum апnиum & 0.03 & - \\
\hline
\end{tabular}

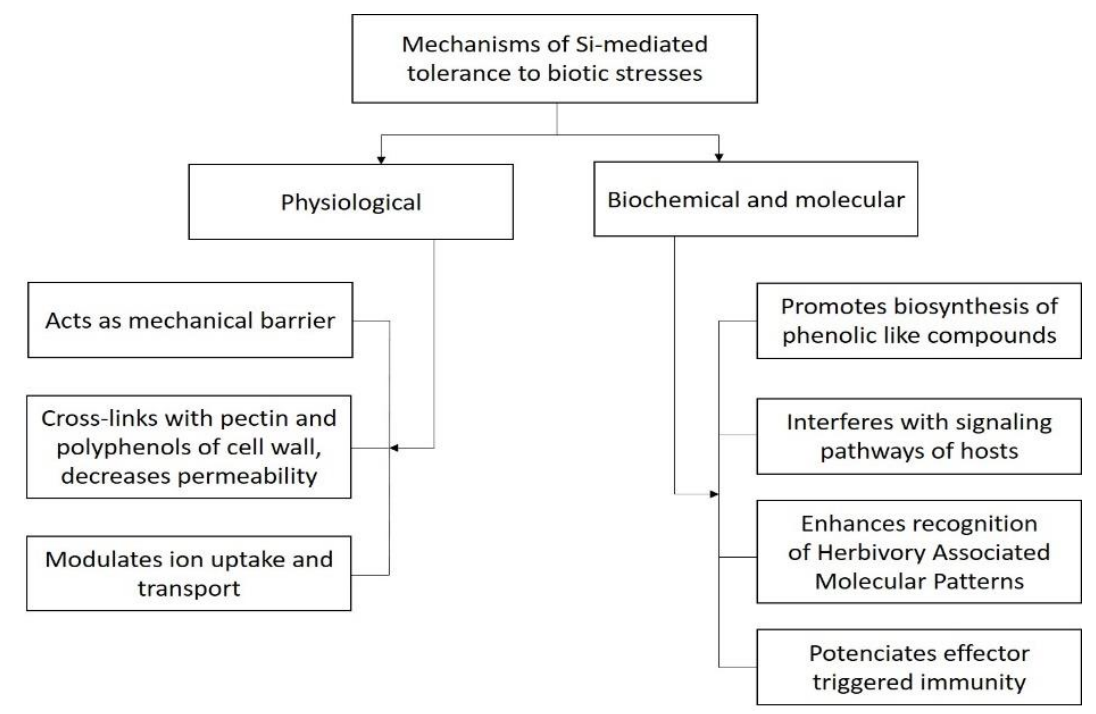

Figure 1. Mechanisms of Si mediated tolerance to biotic stress

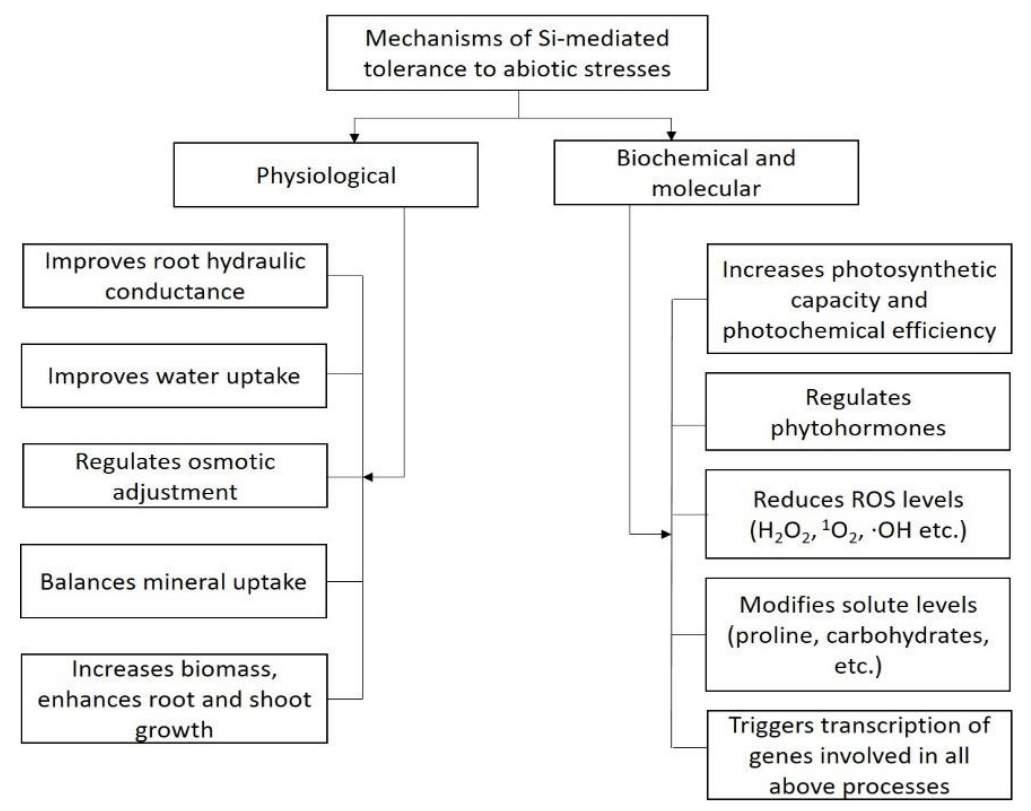

Figure 2. Mechanisms of Si mediated tolerance to abiotic stresses 
Evidently, these are also true with plant-insect (particularly piercing-sucking type) interactions, which involves herbivory associated molecular pattern and effector triggered immunity that mediates plant defense mechanism (Vercelli et al., 2017). Si deposited within the apoplastic space interferes with insects having piercing and sucking type feeding, which showed reduced probing time. The $\mathrm{Si}$ affected extracellular matrix prevented insects from impeding the plant defense response by trapping insect effectors within or by preventing recognition of the plant as a suitable host (Hogenhout \& Bos, 2011). To this date, there are number of reports on $\mathrm{Si}$ mediated resistance against such type of insects including silverleaf whitefly on soybean (Ferreira et al., 2011) and Asian citrus psyllid on lime (Ramírez-Godoy et al., 2018).

\section{Effects of silicon in abiotic stresses}

Abiotic stresses cover a wide range of adverse environmental conditions such as high or low temperature, drought, salinity, ultraviolet radiation and metal toxicity (Epstein, 1999; Ma \& Takahashi, 2002; Ma, 2004). In order to increase food quality and quantity plants need to mobilize different strategies to overcome the losses due to these adverse conditions.

With respect to salinity and drought stress, Si was shown to improve root growth (Kim et al., 2013) and increased water uptake due to improved hydraulic conductance (Wang et al., 2015) and root activity (Chen et al., 2011), which in turn increased root/shoot ratio in Si treated plants (Wang et al., 2015). Further metabolic changes involved modification of solute levels, as was reported for proline (Lee et al., 2010), carbohydrates (Ming et al., 2012), glycine betaine (Torabi et al., 2015), total phenolics (Shi et al., 2014), total soluble sugars and total amino acids (Crusciol et al., 2009). These adjustments may minimize the osmotic shock created by salt and drought exposures. Moreover, Si supply can interfere with hormonal regulatory circuits under diverse stresses (Kim et al., 2013) by decreasing JA (Kim et al., 2013) and enhancing the concentration of endogenous GA (Lee et al., 2010) and salicylic acid (Hamayun et al., 2010). In a noteworthy example, upregulated cytokinin level was associated with silicon treatment in tomato (Barreto et al., 2021) and cucumber (Zhu et al., 2020). In terms of the oxidative component of stresses $\mathrm{Si}$ plays an important role by improving antioxidant ability (Gunes et al., 2007) and regulates the production of polyamines, like putrescine, spermidine and spermine (Liu \& Xu, 2007; Wang et al., 2015). Our own recent research elucidated that $\mathrm{Si}$ could downregulate pro-oxidant systems in plants as well. Lipoxygenases were found downregulated in $\mathrm{Si}$ treated cucumber shoot tissues along with an overall decrease in oxidative stress level (Bat-Erdene et al., 2021). Based on the coordinated attenuation of all lipoxygenases' expression, it was assumed that this regulation may be at least partly responsible for the observed antioxidant effect of silicon, potentially also contributing to the growth promoting effect observed. Under salt stress Si decreased MDA concentration, which is an end product of lipid peroxidation in lemon (Mditshwa et al., 2013) and in grapevine rootstock (Soylemezoglu et al., 2009). Moreover, MDA concentration was positively correlated with $\mathrm{Na}^{+}$ absorption in salt stressed cucumber and negative correlation with $\mathrm{Ca}^{2+}$ and $\mathrm{K}^{+}$absorption in the $\mathrm{Si}$ treated cucumber (Khoshgoftarmanesh et al., 2014).

Under drought condition, excess $\mathrm{Si}$ is deposited under the cuticle forming a second layer which decreases water loss via peristomal transpiration in shoots (Ma et al., 2001; Ma, 2004). In roots $\mathrm{Si}$ accumulates around exodermis and endodermis cells, at sites where $\mathrm{Si}$ transporters are located. Si deposits at these positions were postulated to block apoplastic bypass route of solutes containing toxic ions. Due to this inhibition less $\mathrm{Na}^{+}, \mathrm{Cl}^{-}$ and $\mathrm{Cd}^{2+}$ enter via Casparian strip breaks or underdevelopments, resulting decreased bypass in sap flow. The discrimination against the problematic ions can be more strict at membrane transport, these ions will be less likely to accumulate in sensitive tissues of the shoot to a toxic level (Shi et al., 2005; Zuccarini, 2008; Savvas et al., 2009; Flam-Shepherd et al., 2018). Si also stimulates to the formation of the Casparian strip by promoting suberin and lignin biosynthesis, which further protects plants against toxicants through the bypass apoplastic route (Fleck et al., 2015).

Like most environmental stresses, ultraviolet radiation also induces reactive oxygen species production in plants (Shen et al., 2010; Rybus-Zając \& Kubiś, 2010; Beckmann et al., 2012). According to $\mathrm{Li}$ et al. (2008) $\mathrm{Si}$ is shown to increase plant tolerance to UV-B radiation, which has adverse effects on plant growth. Shen et al. (2010) reported that $\mathrm{Si}$ application significantly reduced the membrane damage caused by combination of drought and UV-B radiation in soybean seedlings.

Extreme temperatures also frequently limit plant growth, development and reproduction. When plants are subjected to high or low temperature, cell membrane permeability increases. Under these conditions increased ROS generation is common, including more intense lipid peroxidation, which results from imbalance between production and scavenging (Jaleel et al., 2009). Hu et al., (2020) reported that Si supply during high temperature $\left(40{ }^{\circ} \mathrm{C}\right)$ stress kept stomata open in poinsettia plants. In the same study enhanced resistance to cold stress was reported, which was explained through increased epicuticular wax deposition when the plants were exposed to chilling $\left(4^{\circ} \mathrm{C}\right)$ temperatures.

In the apoplast, $\mathrm{H}_{4} \mathrm{SiO}_{4}$ polymerization results in an amorphous silica barrier (Exley, 2015), which can limit intercellular penetration of toxicants like $\mathrm{Al}, \mathrm{Cd}, \mathrm{Mn}$ and $\mathrm{Zn}$ and their uptake into the symplast (Guerriero et al., 2016). Apoplastic uptake of metal ions may also be reduced by $\mathrm{Si}$ depositions along with a decrease in free metal mobility (Iwasaki et al., 2002; Rogalla \& Römheld, 2002). This effect can be accelerated by enhanced adsorption of metals in the cell wall of root tissues (Liang et al., 2006; Ye et al., 2012). Transport of metals can also be restricted through the endodermis as a result of $\mathrm{Si}$ accumulation. Porosity of cell wall in the inner root tissues may also be reduced, thus decreasing metal concentration in the xylem sap. (Keller et al., 2015). Limited transfer of metals through endodermal cells and decreased ionic mobility in root tissues are the main mechanisms of reducing adverse effects of metal toxicity stress by Si (Etesami \& Jeong, 2018). In other studies, however, effects of silicon on the uptake of micronutrients were found variable in tomato, depending on the genotype and mineral concentration (Kleiber et al., 2015). Effects are probably dependent on the transport process of the minerals, including compartmentalization. Whereas, in cucumber Si supply greatly decreased the phenolic compounds and lipid peroxidation caused by excess Mn (Shi et al., 2005; Maksimović et al., 2007). Different results may also stem from the use of unrelated species, growth and treatment conditions. Interestingly, even in stress free conditions, $\mathrm{Si}$ treatments have shown to improve growth and appearance of various horticultural plants, which also resulted in greater yield (Fleck et al., 2011; Toresano-Sánchez et al., 2012; Fleck et al., 2015; BatErdene et al., 2021) 
Table 2. Literature summary on effects of Si in biotic stress

\begin{tabular}{|c|c|c|c|c|c|c|}
\hline Stress type & $\begin{array}{l}\text { Common name } \\
\text { of } \\
\text { disease/insect }\end{array}$ & $\begin{array}{l}\text { Pathogenic } \\
\text { fungal/insect } \\
\text { species }\end{array}$ & Host plant \& species & Si concentration & $\begin{array}{l}\text { Si effects and symptoms on host } \\
\text { plant }\end{array}$ & References \\
\hline \multirow{11}{*}{ Disease } & \multirow{9}{*}{$\begin{array}{l}\text { Powdery } \\
\text { mildew }\end{array}$} & $\begin{array}{l}\text { Blumeria } \\
\text { graminis }\end{array}$ & $\begin{array}{l}\text { Barley } \\
\text { (Hordeum vulgare L.) } \\
\text { Oat } \\
\text { (Avena sativa) }\end{array}$ & K-silicate & $\begin{array}{l}\text { Mechanical barrier } \\
+ \text { resistance }\end{array}$ & Wagner, 1940 \\
\hline & & \multirow[t]{2}{*}{$\begin{array}{l}\text { Erysiphe } \\
\text { cichoracearu }\end{array}$} & $\begin{array}{l}\text { Cucumber } \\
\text { (Cucumis sativus) }\end{array}$ & $\begin{array}{l}20 \mathrm{mg} \\
\text { Ca-silicate } \\
15 \text { gm } \\
\text { Na-silicate }\end{array}$ & $\begin{array}{l}+ \text { resistance } \\
\text { Prolonged incubation }\end{array}$ & \\
\hline & & & Arabidopsis thaliana & $\begin{array}{l}100 \mathrm{ppm} \\
\text { K-silicate } \\
\end{array}$ & Production of fungitoxic substances & $\begin{array}{l}\text { Ghanmi et al., } \\
2004\end{array}$ \\
\hline & & $\begin{array}{l}\text { Sphaerothecafuligi } \\
\text { nea }\end{array}$ & $\begin{array}{l}\text { Cucumber } \\
\text { (Cucumis sativus L.) }\end{array}$ & $\begin{array}{l}1.7 \mathrm{mM} \\
(100 \mathrm{ppm}) \\
\text { K-silicate }\end{array}$ & $\begin{array}{l}\text { - fungal growth } \\
\text { - hyphal length } \\
\text { + Flavonoid phytoalexins }\end{array}$ & $\begin{array}{l}\text { Samuels et al., } \\
1991 \\
\text { Fawe et al., } \\
1998\end{array}$ \\
\hline & & \multirow{3}{*}{$\begin{array}{l}\text { Podosphaera } \\
\text { xanthii }\end{array}$} & \multirow{2}{*}{$\begin{array}{l}\text { Zucchini } \\
\text { (Cucurbita pepo L.) }\end{array}$} & $\begin{array}{l}0.1 \mathrm{mM} \\
1 \mathrm{mM} \\
\end{array}$ & - expansion of powdery mildew & $\begin{array}{l}\text { Savvas et al., } \\
2009\end{array}$ \\
\hline & & & & 100 ppm & $\begin{array}{l}\text { + chlorophyll content } \\
\text { - disease incident } \\
\text { - disease severity } \\
\end{array}$ & $\begin{array}{l}\text { Vercelli et al., } \\
2017\end{array}$ \\
\hline & & & $\begin{array}{l}\text { Melon } \\
\text { (Cucumis melo) }\end{array}$ & $\begin{array}{l}2 \mathrm{mM} \\
\mathrm{K} \text {-silicate }\end{array}$ & $\begin{array}{l}\text { - disease severity } \\
\text { - disease progress rate } \\
\text { - conidial production } \\
\text { - infection efficiency } \\
\text { - colony area }\end{array}$ & $\begin{array}{l}\text { Dallagnol et } \\
\text { al., } 2012\end{array}$ \\
\hline & & $\begin{array}{l}\text { Podosphaera } \\
\text { aphanis }\end{array}$ & $\begin{array}{l}\text { Strawberry } \\
\text { (Fragaria L.) }\end{array}$ & $0.003 \mathrm{mg} / \mathrm{ml}$ & $\begin{array}{l}\text { + plant resilience } \\
\text { - disease severity } \\
\text { - infection rate } \\
\text { - AUDPC }\end{array}$ & Liu et al., 2020 \\
\hline & & $\begin{array}{l}\text { Oidium } \\
\text { neolycopersici }\end{array}$ & $\begin{array}{l}\text { Tomato (Solanum } \\
\text { lycopersicum) }\end{array}$ & $100 \mathrm{mg} / \mathrm{L}$ & $\begin{array}{l}\text { - disease incidence } \\
\text { - disease severity }\end{array}$ & $\begin{array}{l}\text { Garibaldi et } \\
\text { al., 2011 }\end{array}$ \\
\hline & $\begin{array}{l}\text { Black leg of } \\
\text { seedlings }\end{array}$ & Pythium ultimum & $\begin{array}{l}\text { Cucumber } \\
\text { (Cucumis sativus L.) }\end{array}$ & $\begin{array}{l}1.7 \mathrm{mM} \\
\mathrm{K} \text {-silicate }\end{array}$ & $\begin{array}{l}\text { - wilting } \\
\text { - root decay } \\
\text { - senescence }\end{array}$ & $\begin{array}{l}\text { Chérif et al., } \\
1992\end{array}$ \\
\hline & $\begin{array}{l}\text { Soybean root } \\
\text { rot }\end{array}$ & $\begin{array}{l}\text { Phytophthora } \\
\text { sojae }\end{array}$ & $\begin{array}{l}\text { Soybean (Glycine max } \\
\text { L.) }\end{array}$ & $1.7 \mathrm{mM}$ & $\begin{array}{l}\text { Apoplast interaction } \\
\text { - release of effectors }\end{array}$ & $\begin{array}{l}\text { Wang \& } \\
\text { Wang, 2018 } \\
\text { Rasoolizadeh } \\
\text { et al., } 2018 \\
\end{array}$ \\
\hline \multirow{3}{*}{ Insect } & $\begin{array}{l}\text { Two-spotted } \\
\text { spider mite }\end{array}$ & $\begin{array}{l}\text { Tetranychus } \\
\text { urticae }\end{array}$ & $\begin{array}{l}\text { Strawberry } \\
(\text { Fragaria L.) }\end{array}$ & $0.003 \mathrm{mg} / \mathrm{ml}$ & $\begin{array}{l}\text { - AUPPC } \\
\text { - number of insects per leaf }\end{array}$ & Liu et al., 2020 \\
\hline & $\begin{array}{l}\text { Asian citrus } \\
\text { psyllid }\end{array}$ & Diaphorina citri & $\begin{array}{l}\text { Persian lime } \\
\text { (Citrus latifolia) }\end{array}$ & $\begin{array}{l}2 \mathrm{ml} / \mathrm{L} \\
\mathrm{K} \text {-silicate }\end{array}$ & $\begin{array}{l}\text { - eggs } \\
+ \text { plant resistance }\end{array}$ & $\begin{array}{l}\text { Ramírez- } \\
\text { Godoy et al., } \\
2018\end{array}$ \\
\hline & $\begin{array}{l}\text { Silverleaf } \\
\text { whitefly }\end{array}$ & Bemisia tabaci & $\begin{array}{l}\text { Soybean (Glycine } \max \\
\text { L.) }\end{array}$ & $\begin{array}{l}250 \mathrm{ml} \text { of a } 1 \% \\
\text { silicic acid }\end{array}$ & $\begin{array}{l}\text { - phenolic compounds } \\
\text { + nitrogen production } \\
\text { + resistance } \\
\text { - insect population }\end{array}$ & $\begin{array}{l}\text { Ferreira et al., } \\
2011\end{array}$ \\
\hline
\end{tabular}

+ stands for increased, improved or enhanced

- stands for decreased, alleviated or mitigated

Abbreviations: AUDPC-Area Under the Disease Progress Curve; AUPPC-area under the pathogen progress curve.

Table 3. Literature summary on effects of $\mathrm{Si}$ in abiotic stresses

\begin{tabular}{|c|c|c|c|c|}
\hline Stress & Species & $\begin{array}{l}\text { Si concentration } \\
\text { applied }\end{array}$ & Effects of $\mathrm{Si}$ & References \\
\hline \multirow{4}{*}{ Salinity } & \multirow{2}{*}{$\begin{array}{l}\text { Rice } \\
(\text { Oryza sativa })\end{array}$} & $\begin{array}{l}0.5 \mathrm{mM} \\
1.0 \mathrm{mM} \\
2.0 \mathrm{mM} \\
\end{array}$ & $\begin{array}{l}+ \text { ABA tissue concentration } \\
-\mathrm{JA} \\
+\mathrm{SA} \text { biosynthesis }\end{array}$ & Kim et al., 2013 \\
\hline & & $3 \mathrm{mM}$ & $\begin{array}{l}+ \text { Shoot growth } \\
\text { - Apoplastic transport of } \mathrm{Na}^{+} \\
\text {- bypass flow }\end{array}$ & Flam-Shepherd et al., 2018 \\
\hline & \multirow[t]{2}{*}{$\begin{array}{l}\text { Cucumber } \\
\text { (Cucumis sativus) }\end{array}$} & $0.83 \mathrm{mM}$ & $\begin{array}{l}+ \text { root hydraulic conductance } \\
+\mathrm{K}^{+} \\
-\mathrm{Na}^{+}\end{array}$ & Wang et al., 2015 \\
\hline & & $1 \mu \mathrm{M}$ & - Lipid peroxidation & Khoshgoftarmanesh et al., 2014 \\
\hline
\end{tabular}




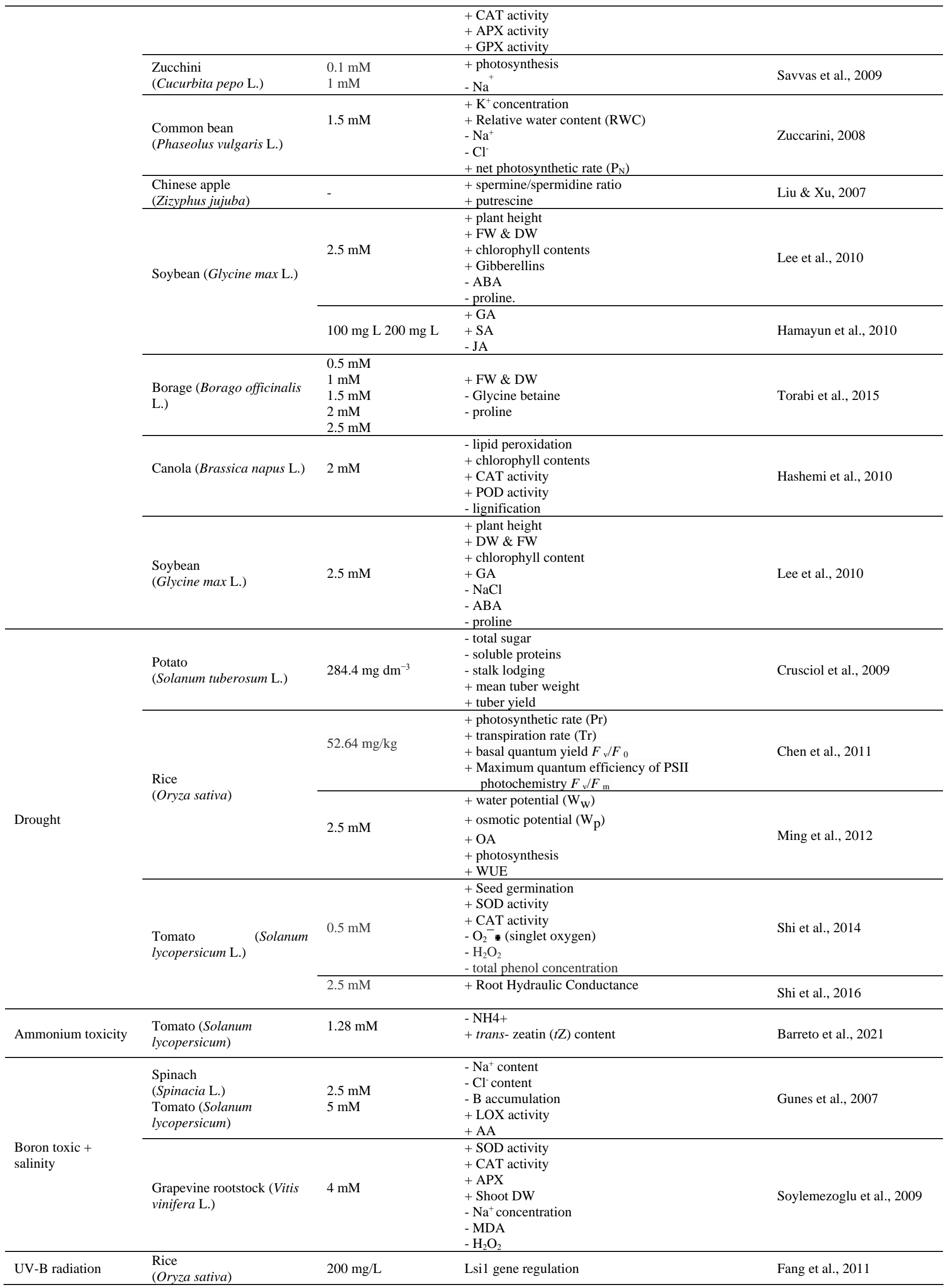




\begin{tabular}{|c|c|c|c|c|}
\hline $\begin{array}{l}\text { UV-B + } \\
\text { drought }\end{array}$ & Soybean (Glycine $\max$ L.) & $1.7 \mathrm{mM}$ & $\begin{array}{l}\text { + relative leaf water content } \\
+ \text { Anthocyanin level } \\
\text { + phenol level } \\
+ \text { photosynthesis }\end{array}$ & Shen et al., 2010 \\
\hline \multirow[t]{2}{*}{ Temperature stress } & $\begin{array}{l}\text { Tomato (Solanum } \\
\text { lycopersicum) }\end{array}$ & $75 \mathrm{mg} / \mathrm{L}$ & $\begin{array}{l}\text { + stomatal closure } \\
\text { + epicuticular wax } \\
\text { + photosynthetic rate } \\
\text { - MDA } \\
\text { + hydrogen sulfide } \\
\text { + APX activity }\end{array}$ & Hu et al., 2020 \\
\hline & $\begin{array}{l}\text { Lemon } \\
\text { (Citrus L.) }\end{array}$ & $\begin{array}{l}50 \mathrm{mg} / \mathrm{L} \\
150 \mathrm{mg} / \mathrm{L} \\
250 \mathrm{mg} / \mathrm{L}\end{array}$ & $\begin{array}{l}\text { - MDA } \\
+ \text { total antioxidants } \\
\text { - total phenolics }\end{array}$ & Mditshwa et al., 2013 \\
\hline \multirow[b]{3}{*}{ Mn toxicity } & $\begin{array}{l}\text { Tomato } \\
\text { (Solanum lycopersicum) }\end{array}$ & $0.3 \mathrm{mg} \mathrm{dm}^{3}$ & $\begin{array}{l}\text { + Microelement level } \\
\text { + photosynthesis }\end{array}$ & Kleiber et al., 2015 \\
\hline & & $\begin{array}{l}0.5 \mu \mathrm{M} \\
50 \mu \mathrm{M} \\
100 \mu \mathrm{M} \\
\end{array}$ & $\begin{array}{l}\text { + biomass production } \\
\text { - phenolic compounds } \\
\text { No symptoms of Mn toxicity }\end{array}$ & Maksimović et al., 2007 \\
\hline & $\begin{array}{l}\text { Cucumber } \\
\text { (Cucumis sativus) }\end{array}$ & $1 \mathrm{mM}$ & $\begin{array}{l}\text { + SOD activity } \\
\text { + APX activity } \\
\text { + DHAR activity } \\
\text { + GR activity } \\
\text { + Ascorbate concentration } \\
\text { + GSH concentration } \\
\text { - lipid peroxidation }\end{array}$ & Shi et al., 2005 \\
\hline $\begin{array}{l}\mathrm{K}^{+} \\
\text {deficiency }\end{array}$ & $\begin{array}{l}\text { Maize } \\
\text { (Zea mays L.) }\end{array}$ & $2 \mathrm{mM}$ & $\begin{array}{l}\text { Nutritional } \\
\text { Physiological } \\
\text { Growth variables }\end{array}$ & $\begin{array}{l}\text { dos Santos Sarah et al., } \\
2021\end{array}$ \\
\hline \multirow{3}{*}{ No stress condition } & $\begin{array}{l}\text { Rice } \\
\text { (Oryza sativa) } \\
\text { Maize } \\
\text { (Zea mays) } \\
\text { Onion } \\
(\text { Allium cepa) } \\
\end{array}$ & $\begin{array}{l}1.78 \mathrm{mM} \\
1.07 \mathrm{mM}\end{array}$ & $\begin{array}{l}\text { Casparian band formation } \\
+ \text { phenol complex }\end{array}$ & Fleck et al., 2015 \\
\hline & $\begin{array}{l}\text { Cucumber (Cucumis } \\
\text { sativus) }\end{array}$ & $1.67 \mathrm{mM}$ & $\begin{array}{l}\text { + Shoot growth } \\
\text { - MDA } \\
-\mathrm{H}_{2} \mathrm{O}_{2} \\
\text { - LOX }\end{array}$ & Bat-Erdene et al., 2021 \\
\hline & $\begin{array}{l}\text { Cherry tomato } \\
\text { (Lycopersicon } \\
\text { esculentum var. } \\
\text { cerasiforme) }\end{array}$ & $250 \mathrm{~mL} / \mathrm{ha}$ & $\begin{array}{l}+ \text { fruit } \\
+ \text { yield }\end{array}$ & $\begin{array}{l}\text { Toresano-Sánchez et al., } \\
2012\end{array}$ \\
\hline
\end{tabular}

+ stands for increased, improved or enhanced

- stands for decreased, alleviated or mitigated

Abbreviations: AA-antioxidant activity; ABA-Abscisic acid; APX-Ascorbate peroxidase; CAT-catalase; FW-fresh weight, DR-dry weight; DHAR-dehydroascorbate reductase; GA-gibberellins; GR-Glutathione reductase; GSH-glutathione; JA-Jasmonic acid; LOX-lipoxygenase; MDA-Malondialdehyde; POD-peroxidase; SAsalicylic acid; SOD-superoxide dismutase; WUE-water use efficiency.

\section{Conclusion}

In recent years a growing number of transporters have been identified that mediate $\mathrm{Si}$ uptake and transport in plants and various molecular studies have been conducted on their expression and activities. However, it is still a great challenge ahead to dig deeper into their regulation as well as to fully understand their function. Hence, transcriptional regulation of $\mathrm{Si}$ transporter genes and transmembrane $\mathrm{Si}$ transport needs further investigations. Apparently, only limited characterization of $\mathrm{Si}$ transporters is available at present in a few plant species, including horticultural crops. Clearly more research is needed for a deeper understanding of the physiological significance of Si transporters in different plants. This will facilitate breeding programs for exploiting most potential benefits of $\mathrm{Si}$ accumulation in crops.

As for the molecular mechanisms to explain the diverse effects silicon can exert in plants a unifying concept has been put forward. The "apoplastic obstruction hypothesis" postulates that most, if not all, effects of extra silicon supplementation are attributable to extracellular accumulation of Si depositions and derive from disturbed physiological events there with diverse downstream consequences (Coskun et al., 2019). In view of most above examples and that of our own results we agree with this conclusion.

In practical application, Si fertilizers have been spreading in cultivation of horticultural crops for improved performance and protection against adverse environmental effects. Their larger scale application requires precise information about $\mathrm{Si}$ effects in specific genotypes, media and treatment regimes. To meet this demand more laboratory and field trials are required in order to ensure high quality products and safe harvest in crop production.

\section{Acknowledgements}

This research was supported by the Ministry of Innovation and Technology within the framework of the Thematic Excellence Program 2020, Institutional Excellence SubProgram (TKP2020-IKA-12) in the topic of water-related researches of Szent István University/Hungarian University of Agriculture and Life Sciences.

\section{References}

Adrees, M., Ali, S., Rizwan, M., Zia-Ur-Rehman, M., Ibrahim, M., Abbas, F., Farid, M., Qayyum, M. F., Irshad, 
M. K. (2015): Mechanisms of silicon-mediated alleviation of heavy metal toxicity in plants: A review. Ecotoxicology Environmental Safety 119: 186-197. https://doi.org/10.1016/j. ecoenv.2015.05.011

Ahmed, M., Qadeer, U., Aslam, M. (2011): Silicon application and drought tolerance mechanism of sorghum. African Journal of Agricultural Research. 6: 594-607.

Bakhat, H. F., Hanstein, S., Schubert, S. (2009): Optimal level of silicon for maize (Zea mays L. c.v. AMADEO) growth in nutrient solution under controlled conditions.

Barber, D. A., Shone, M. G. T. (1967): The Initial Uptake of Ions by Barley Roots: III. The uptake of cations. Journal of Experimental Botany. 18: 631-643.

Barreto, R. F., de Mello Prado, R., Lúcio, J. C. B., LópezDíaz, I., Carrera, E. and Carvalho, R. F. (2021): Ammonium toxicity alleviation by silicon is dependent on cytokinins in tomato cv. Micro-Tom. Journal of Plant Growth Regulation. 1-12. https://doi.org/10.1007/s00344-021-10314-5

Bat-Erdene, O., Szegő, A., Gyöngyik, M., Mirmazloum, I., Papp, I. (2021): Long term silicon exposure coordinately downregulates lipoxygenase genes, decreases reactive oxygen species level and promotes growth of cucumber plants in a semi-hydroponic cultivation system. Russian Journal of Plant Physiology.68(5)

Bauer, P., Elbaum, R., Weiss, I. M. (2011): Calcium and silicon mineralization in land plants: transport, structure and function. Plant Science: International Journal of Experimental Plant Biology. 180: 746-756. https://doi.org/10.1016/ j.plantsci.2011.01.019

Beckmann, M., Hock, M., Bruelheide, H., Erfmeier, A. (2012): The role of UV-B radiation in the invasion of Hieracium pilosella-A comparison of German and New Zealand plants. Environmental and Experimental Botany 75: 173-180. https://doi.org/10.1016/j.envexpbot.2011.09.010

Bienert, G. P., Schüssler, M. D., Jahn, T. P. (2008): Metalloids: essential, beneficial or toxic? Major intrinsic proteins sort it out. Trends in Biochemical Sciences. 33: 20-26. https://doi.org/10.1016/j.tibs.2007.10.004

Bozkurt, T. O., Schornack, S., Banfield, M. J., Kamoun, S. (2012): Oomycetes, effectors, and all that jazz. Current Opinion in Plant Biology 15: 483-492. https://doi.org/10.1016/j.pbi.2012.03.008

Chen, W., Yao, X., Cai, K., Chen, J. (2011): Silicon alleviates drought stress of rice plants by improving plant water status, photosynthesis and mineral nutrient absorption. Biological Trace Element Research. 142: 67-76. https://doi.org/10.1007/s12011-010-8742-X

Chérif, M., Menzies, J. G., Benhamou, N., Bélanger, R. R. (1992): Studies of silicon distribution in wounded and Pythium ultimum infected cucumber plants. Physiological and Molecular Plant Pathology. 41: 371-385. https://doi.org/10.1016/0885-5765(92)90022-N

Chiba, Y., Mitani, N., Yamaji, N., Ma, J. F. (2009): HvLsi1 is a silicon influx transporter in barley. The Plant Journal for Cell and Molecular Biology. 57: 810-818. https://doi.org/10.1111/j.1365-313X.2008.03728.x

Coskun, D., Deshmukh, R., Sonah, H., Menzies, J. G., Reynolds, O., Ma, J. F., Kronzucker, H. J., Bélanger, R. R. (2019): The controversies of silicon's role in plant biology. New Phytologist. 221(1): 67-85. DOI: 10.1111/nph.15343

Crusciol, C. A. C., Pulz, A. L., Lemos, L. B., Soratto, R. P., Lima, G. P. P. (2009): Effects of silicon and drought stress on tuber yield and leaf biochemical characteristics in potato. Crop Science. 49(3): 949-954. https://doi.org/10.2135/cropsci2008. 04.0233

Dallagnol, L. J., Rodrigues, F. A., Tanaka, F. A. O., Amorim, L., Camargo, L. E. A. (2012): Effect of potassium silicate on epidemic components of powdery mildew on melon. Plant Pathology. 61(2): 323-330. https://doi.org/10.1111/ j.1365-3059.2011.02518.x

Datnoff, L. E., Snyder, G. H., Korndörfer, G. H. (2001): Silicon in Agriculture. Elsevier.

Deshmukh, R., Bélanger, R.R. (2016): Molecular evolution of aquaporins and silicon influx in plants. Functional Ecology. 30: 1277-1285. https://doi.org/10.1111/1365-2435.12570

Deshmukh, R. K., Vivancos, J., Guérin, V., Sonah, H., Labbé, C., Belzile, F., Bélanger, R. R. (2013): Identification and functional characterization of silicon transporters in soybean using comparative genomics of major intrinsic proteins in Arabidopsis and rice. Plant Molecular Biology. 83: 303-315. https://doi.org/10.1007/s11103-013-0087-3

Epstein, E. (1999): Silicon. Annual Review of Plant Physiology and Plant Molecular Biology. 50: 641-664. https://doi.org/10.1146/annurev.arplant.50.1.641

Epstein, E. (1994): The anomaly of silicon in plant biology. Proceedings of the National Academy of Sciences of the U. S. A. 91: 11-17. https://doi.org/10.1073/pnas.91.1.11

Etesami, H., Jeong, B. R. (2018): Silicon (Si): Review and future prospects on the action mechanisms in alleviating biotic and abiotic stresses in plants. Ecotoxicology and Environmental Safety. 147: 881-896. https://doi.org/10.1016/j.ecoenv.2017.09.063

Exley, C. (2015): A possible mechanism of biological silicification in plants. Frontiers in Plant Science. 6. https://doi.org/10.3389/fpls.2015.00853

Faiyue, B., Vijayalakshmi, C., Nawaz, S., Nagato, Y., Taketa, S., Ichii, M., Al-Azzawi, M. J., Flowers, T. J. (2010): Studies on sodium bypass flow in lateral rootless mutants lrt1 and lrt2, and crown rootless mutant crl1 of rice (Oryza sativa L.). Plant Cell and Environment. 33: 687-701. https://doi.org/10.1111/j.1365-3040.2009.02077.x

Farooq, M. A., Dietz, K. J. (2015): Silicon as Versatile Player in Plant and Human Biology: Overlooked and Poorly Understood. Frontiers in Plant Science. 6: 994.

Fawe, A., Abou-Zaid, M., Menzies, J. G., Bélanger, R. R. (1998): Silicon-mediated accumulation of flavonoid phytoalexins in cucumber. Phytopathology. 88: 396-401. https://doi.org/10.1094/PHYTO.1998.88.5.396

Ferreira, R. S., Moraes, J. C., Antunes, C. S. (2011): Silicon influence on resistance induction against Bemisia tabaci biotype B (Genn.) (Hemiptera: Aleyrodidae) and on vegetative development in two soybean cultivars. Neotropical entomology. 40(4): 495-500. https://doi.org/10.1590/S1519566X2011000400014

Flam-Shepherd, R., Huynh, W. Q., Coskun, D., Hamam, A. M., Britto, D. T., Kronzucker, H. J. (2018): Membrane fluxes, bypass flows, and sodium stress in rice: the influence of 
silicon. Journal of Expimental Botany. 69: 1679-1692. https://doi.org/10.1093/jxb/erx460

Fleck, A. T., Schulze, S., Hinrichs, M., Specht, A., Waßmann, F., Schreiber, L., Schenk, M. K. (2015): Silicon Promotes Exodermal Casparian Band Formation in SiAccumulating and Si-Excluding Species by Forming Phenol Complexes. PloS One. 10: e0138555. https://doi.org/10.1371/journal.pone.0138555

Ghanmi, D., McNally, D., Benhamou, N., Menzies, J., Belanger, R. (2004): Powdery mildew of Arabidopsis thaliana: A pathosystem for exploring the role of silicon in plantmicrobe interactions. Physiological and Molecular Plant Pathology. 64: 189-199. https://doi.org/10.1016/j.pmpp. 2004.07.005

Giraldo, M. C., Valent, B. (2013): Filamentous plant pathogen effectors in action. Nature Reviews Microbiology. 11: 800-814. https://doi.org/10.1038/nrmicro3119

Gomes, D., Agasse, A., Thiébaud, P., Delrot, S., Gerós, H., Chaumont, F. (2009): Aquaporins are multifunctional water and solute transporters highly divergent in living organisms. Biochimica et Biophysica Acta. 1788: 1213-1228. https://doi.org/10.1016/j.bbamem.2009.03.009

Gong, H. J., Randall, D. P., Flowers, T. J. (2006): Silicon deposition in the root reduces sodium uptake in rice (Oryza sativa L.) seedlings by reducing bypass flow. Plant Cell and Environment. 29: 1970-1979. https://doi.org/10.1111/j.13653040.2006.01572.x

Guerriero, G., Hausman, J. F., Legay, S. (2016): Silicon and the Plant Extracellular Matrix. Frontiers in Plant Science. 7: 463. https://doi.org/10.3389/fpls.2016.00463

Gunes, A., Inal, A., Bagci, E. G., Pilbeam, D. J., (2007): Silicon-mediated changes of some physiological and enzymatic parameters symptomatic for oxidative stress in spinach and tomato grown in sodic-B toxic soil. Plant and Soil. 290(1): 103-114. https://doi.org/10.1007/s11104-006-9137-9

Hamayun, M., Sohn, E. Y., Afzal Khan, S., Shinwari, Z., Khan, A., Lee, I. J. (2010): Silicon alleviates the adverse effects of salinity and drought stress on growth and endogenous plant growth hormones of soybean (Glycine max L.). Pakistan Journal of Botany. 42(3): 1713-1722.

Hattori, T., Sonobe, K., Araki, H., Inanaga, S., An, P., Morita, S. (2008): Silicon Application by Sorghum Through the Alleviation of Stress-Induced Increase in Hydraulic Resistance. Journal of Plant Nutrition. 31: 1482-1495. https://doi.org/10.1080/01904160802208477

Hodson, M. J., White, P. J., Mead, A. and Broadley, M. R. (2005): Phylogenetic variation in the silicon composition of plants. Annals of botany. 96(6): 1027-1046. https://doi.org/ 10.1093/aob/mci255

Hogenhout, S. A., Bos, J. I. B. (2011): Effector proteins that modulate plant--insect interactions. Current Opinion in Plant Biology. 14: 422-428. https://doi.org/10.1016/j.pbi. 2011.05.003

Holub, E. B., Cooper, A. (2004): Matrix, reinvention in plants: how genetics is unveiling secrets of non-host disease resistance. Trends in Plant Science. 9: 211-214. https://doi.org/10.1016/j.tplants.2004.03.002

Horiguchi, T. (1988): Mechanism of manganese toxicity and tolerance of plants. Soil Science and Plant Nutrition. 34: 6573. https://doi.org/10.1080/00380768.1988.10415580
Hu, J., Li, Y., Jeong, B. (2020): Silicon Alleviates Temperature Stresses in Poinsettia by Regulating Stomata, Photosynthesis, and Oxidative Damages. Agronomy. 10. https://doi.org/10.3390/agronomy10091419

Iwasaki, K., Maier, P., Fecht, M., Horst, W. (2002): Leaf apoplastic silicon enhances manganese tolerance of cowpea (Vigna unguiculata). Journal of Plant Physiology. 159: 167 173. https://doi.org/10.1078/0176-1617-00691

Jaleel, C.A., Riadh, K., Gopi, R., Manivannan, P., Inès, J., Al-Juburi, H.J., Chang-Xing, Z., Hong-Bo, S., Panneerselvam, R. (2009): Antioxidant defense responses: physiological plasticity in higher plants under abiotic constraints. Acta Physiologiae Plantarum. 31: 427-436. https://doi.org/10.1007/s11738-009-0275-6

Jarvis, S. C. (1987): The effects of low, regulated supplies of nitrate and ammonium nitrogen on the growth and composition of perennial ryegrass, in: Van Diest, A. (Ed.), Plant and Soil Interfaces and Interactions: Proceedings of the International Symposium: Plant and Soil: Interfaces and Interactions. Wageningen, The Netherlands August 6-8, 1986, Developments in Plant and Soil Sciences. Springer Netherlands, Dordrecht, pp. 99-112. https://doi.org/10.1007/978-94-009-3627-0_7

Keller, C., Rizwan, M., Davidian, J.-C., Pokrovsky, O.S., Bovet, N., Chaurand, P., Meunier, J. D. (2015): Effect of silicon on wheat seedlings (Triticum turgidum L.) grown in hydroponics and exposed to 0 to $30 \mu \mathrm{M} \mathrm{Cu}$. Planta. 241; 847860 .

Khalid, R. A., Silva, J. A. (1980): Residual effect of calcium silicate on $\mathrm{Ph}$, phosphorus, and aluminum in a tropical soil profile. Soil Science and Plant Nutrition. 26: 87-98. https://doi.org/10.1080/00380768.1980.10433215

Khoshgoftarmanesh, A. H., Khodarahmi, S., Haghighi, M. (2014): Effect of silicon nutrition on lipid peroxidation and antioxidant response of cucumber plants exposed to salinity stress. Archives of Agronomy and Soil Science. 60: 639-653. https://doi.org/10.1080/03650340.2013.822487

Kim, Y. H., Khan, A., Waqas, M., Shim, J. K., Kim, D. Lee, K. Y., Lee, I. (2013): Silicon Application to Rice Root Zone Influenced the Phytohormonal and Antioxidant Responses Under Salinity Stress. Journal of Plant Growth Regulation. 33. https://doi.org/10.1007/s00344-013-9356-2

Kleiber, T., Calomme, M. and Borowiak, K. (2015): The effect of choline-stabilized orthosilicic acid on microelements and silicon concentration, photosynthesis activity and yield of tomato grown under Mn stress. Plant Physiology and Biochemistry. 96: 180-188. https://doi.org/10.1016/j.plaphy. 2015.07.033

Lee, S., Sohn, E., Hamayun, M., Yoon, J., Lee, I. (2010): Effect of silicon on growth and salinity stress of soybean plant grown under hydroponic system. Agroforestry Systems. 80: 333-340. https://doi.org/10.1007/s10457-010-9299-6

Li, B., Wei, A., Song, C., Li, N., Zhang, J. (2008): Heterologous expression of the TsVP gene improves the drought resistance of maize. Plant Biotechnology Journal. 6: 146-159. https://doi.org/10.1111/j.1467-7652.2007.00301.x

Liang, Y., Chen, Q., Liu, Q., Zhang, W., Ding, R. (2003): Exogenous Silicon ( $\mathrm{Si}$ ) Increases Antioxidant Enzyme Activity and Reduces Lipid Peroxidation in Roots of Salt-Stressed Barley (Hordeum vulgare L.). Journal of Plant Physiology. 160: 1157-64. https://doi.org/10.1078/0176-1617-01065 
Liang, Y., Hua, H., Zhang, J., Cheng, C., Römheld, V. (2006): Importance of plant species and external silicon concentration to active silicon uptake and transport. New Phytologist. 172: 63-72. https://doi.org/10.1111/j.14698137.2006.01797.x

Liang, Y., Nikolic, M., Bélanger, R., Gong, H., Song, A. (2015): Silicon in Agriculture: From Theory to Practice. Springer Netherlands. https://doi.org/10.1007/978-94-0179978-2

Liang, Y., Si, J., Römheld, V. (2005): Silicon uptake and transport is an active process in Cucumis sativus. New Phytologist. 167: 797-804. https://doi.org/10.1111/j.14698137.2005.01463.x

Liang, Y., Sun, W., Christie, P. (2007): Mechanisms of silicon-mediated alleviation of abiotic stresses in higher plants: A review. Environmental Pollution (Barking, Essex: 1987). 147: 422-8. https://doi.org/10.1016/j.envpol.2006.06.008

Liu, B., Davies, K., Hall, A. (2020): Silicon builds resilience in strawberry plants against both strawberry powdery mildew Podosphaera aphanis and two-spotted spider mites Tetranychus urticae. PLOS ONE 15(12). e0241151. https://doi.org/10.1371/journal.pone.0241151

Liu, Y. X., Xu, X. Z. (2007): Effects of Silicon on Polyamine Types and Forms in Leaf of Zizyphus jujube cv. Jinsi-xiaozao Under Salt Stress. Journal of Nanjing Forestry University (Natural Sciences Edition), 4.

Lumsdon, D.G., Farmer, V.C. (1995): Solubility characteristics of proto-imogolite sols: how silicic acid can detoxify aluminium solutions. European Journal of Soil Science. 46: 179-186. https://doi.org/10.1111/j.1365-2389.1995. tb01825.x

Ma, J. F. (2004): Role of silicon in enhancing the resistance of plants to biotic and abiotic stresses. Soil Science and Plant Nutrition. 50: 11-18. https://doi.org/10.1080/00380768.2004. 10408447

Ma, J. F., Goto, S., Tamai, K., Ichii, M. (2001): Role of Root Hairs and Lateral Roots in Silicon Uptake by Rice. Plant Physiology. 127: 1773-1780. https://doi.org/10.1104/pp. 010271

Ma, J. F., Mitani, N., Nagao, S., Konishi, S., Tamai, K., Iwashita, T., Yano, M. (2004): Characterization of the Silicon Uptake System and Molecular Mapping of the Silicon Transporter Gene in Rice. Plant Physiology. 136: 3284-3289. https://doi.org/10.1104/pp.104.047365

Ma, J. F., Takahashi, E. (2002). Soil, fertilizer, and plant silicon research in Japan. Elsevier, Amsterdam; Boston.

Ma, J. F., Yamaji, N. (2006): Silicon uptake and accumulation in higher plants. Trends in Plant Science. 11; 392-397. https://doi.org/10.1016/j.tplants.2006.06.007

Ma, J. F., Yamaji, N. (2015): A cooperative system of silicon transport in plants. Trends in Plant Science. 20(7): 435-442. https://doi.org/10.1016/j.tplants.2015.04.007

Ma, J.F., Yamaji, N., Mitani, N., Tamai, K., Konishi, S., Fujiwara, T., Katsuhara, M., Yano, M. (2007): An efflux transporter of silicon in rice. Nature. 448: 209-212. https://doi.org/10.1038/nature05964

Ma, J. F., Yamaji, N., Mitani-Ueno, N. (2011): Transport of silicon from roots to panicles in plants. Proceedings of the Japan Academy, Series B, Physical and Biological Sciences. 87: 377-385. https://doi.org/10.2183/pjab.87.377
Maksimović, J. D., Bogdanović, J., Maksimović, V., Nikolic, M. (2007): Silicon modulates the metabolism and utilization of phenolic compounds in cucumber (Cucumis sativus L.) grown at excess manganese. Journal of Plant Nutrition and Soil Science. 170(6): 739-744.

Markovich, O., Steiner, E., Kouřil, Š., Tarkowski, P., Aharoni, A. and Elbaum, R. (2017): Silicon promotes cytokinin biosynthesis and delays senescence in Arabidopsis and Sorghum. Plant, Cell \& Environment, 40(7): 1189-1196. https://doi.org/10.1111/pce.12913

Marron, A. O., Ratcliffe, S., Wheeler, G. L., Goldstein, R. E., King, N., Not, F., de Vargas, C., Richter, D. J. (2016): The Evolution of Silicon Transport in Eukaryotes. Molecular Biology and Evolution. 33: 3226-3248. https://doi.org/10.1093/molbev/msw209

Matichenkov, V. V., Ammosova, Y. M. and Bocharnikova, E. A. (1997): The method for determination of plant available silica in soil. Agrochemistry. 1: 76-84.

Matichenkov, V. V. (1990): Amorphous oxide of silicon in soddy podzolic soil and its influence on plants. Canadian Dissertation. 26. Moscow State University, Moscow.

Matychenkov, V. V., Pinskiy, D. L., Bocharnikova, Y. A. (1995): Influence of mechanical compaction of soils on the state and form of available silicon. Eurasian soil science. 27(12): 58-67.

Mditshwa, A., Bower, J. P., Bertling, I., Mathaba, N. (2013): Investigation of the efficiency of the total antioxidants assays in Silicon-treated lemon fruit (Citrus limon). In II All Africa Horticulture Congress. 1007: 93-102. https://doi.org/10.17660/ActaHortic.2013.1007.7

Ming, D. F., Pei, Z. F., Naeem, M. S., Gong, H. J., Zhou, W. J. (2012): Silicon Alleviates PEG-Induced Water-Deficit Stress in Upland Rice Seedlings by Enhancing Osmotic Adjustment. Journal of Agronomy and Crop Science. 198: 14 26. https://doi.org/10.1111/j.1439-037X.2011.00486.x

Mitani, N., Ma, J. F. (2005): Uptake system of silicon in different plant species. Journal of Experimental Botany. 56: 1255-1261. https://doi.org/10.1093/jxb/eri121

Mitani, N., Yamaji, N., Ago, Y., Iwasaki, K., Ma, J. F. (2011): Isolation and functional characterization of an influx silicon transporter in two pumpkin cultivars contrasting in silicon accumulation. Plant Journal of Cell Molecular Biology. 66: 231240. https://doi.org/10.1111/j.1365-313X.2011. 04483.x

Mitra, G. N. (2015): Silicon (Si) Uptake, in: Mitra, G.N. (Ed.), Regulation of Nutrient Uptake by Plants: A Biochemical and Molecular Approach. Springer India, New Delhi, pp. 181-187. https://doi.org/10.1007/978-81-322-2334-4_19

Moussa, H. (2006): Influence of exogenous application of silicon on physiological response of salt-stressed maize (Zea mays L.). International Journal of Agricultural Biology. 8530: 8-2.

Nikolic, M., Nikolic, N., Liang, Y., Kirkby, E.A., Römheld, V. (2007): Germanium-68 as an Adequate Tracer for Silicon Transport in Plants. Characterization of Silicon Uptake in Different Crop Species. Plant Physiology. 143: 495-503. https://doi.org/10.1104/pp.106.090845

Nürnberger, T., Lipka, V. (2005): Non-host resistance in plants: new insights into an old phenomenon. Molecular Plant Pathology. 6: 335-345. https://doi.org/10.1111/j.13643703.2005.00279.x 
Orlov, D. S. (1985): Humus acids of soils. Published for the United States Dept. of Agriculture and the National Science Foundation, Washington, D.C., by Amerind Pub. Co, New Delhi.

Ramírez-Godoy, A., Puentes-Pérez, G., Restrepo-Díaz, H. (2018): An evaluation of the use of calcium, potassium and silicon for the management of Diaphorina citri populations in Tahiti lime trees. Notulae Botanicae Horti Agrobotanici ClujNapoca. 46(2): 546-552. https://doi.org/10.15835/ nbha46211152

Rasoolizadeh, A., Labbé, C., Sonah, H., Deshmukh, R., Belzile, F., Menzies, J., Belanger, R. (2018): Silicon protects soybean plants against Phytophthora sojae by interfering with effector-receptor expression. BMC Plant Biology. 18. https://doi.org/10.1186/s12870-018-1312-7

Raven, J. A. (2001): Chapter 3 Silicon transport at the cell and tissue level, in: Silicon in Agriculture. pp. 41-55. https://doi.org/10.1016/S0928-3420(01)80007-0

Rogalla, H., Römheld, V. (2002): Role of leaf apoplast in silicon-mediated manganese tolerance of Cucumis sativus $\mathrm{L}$. Plant Cell and Environment. 25: 549-555. https://doi.org/10.1046/j.1365-3040.2002.00835.x

Rybus-Zając, M., Kubiś, J. (2010): Effect of UV-B Radiation on Antioxidative Enzyme Activity in Cucumber Cotyledons. Acta Biologica Cracoviensia s. Botanica. 52. https://doi.org/10.2478/v10182-010-0030-8

Samuels, A., Glass, A., Ehret, D., Menzies, J. (1991): Distribution of silicon in cucumber leaves during infection by powdery mildew fungus (Sphaerotheca fuliginea). Canadian Journal of Botany. 69: 140-146. https://doi.org/10.1139/b91-020

Savvas, D., Giotis, D., Chatzieustratiou, E., Bakea, M., Patakioutas, G. (2009): Silicon supply in soilless cultivations of zucchini alleviates stress induced by salinity and powdery mildew infections. Environmental and experimental botany. 65(1): 11-17. https://doi.org/10.1016/j.envexpbot.2008.07.004

Shen, X., Zhou, Y., Duan, L., Li, Z., Eneji, A. E., Li, J. (2010): Silicon effects on photosynthesis and antioxidant parameters of soybean seedlings under drought and ultravioletB radiation. Journal of Plant Physiology. 167: 1248-1252. https://doi.org/10.1016/j.jplph.2010.04.011

Shi, Q., Bao, Z., Zhu, Z., He, Y., Qian, Q., Yu, J. (2005): Silicon-mediated alleviation of Mn toxicity in Cucumis sativus in relation to activities of superoxide dismutase and ascorbate peroxidase. $\quad$ Phytochemistry. 66: 1551-1559. https://doi.org/10.1016/j.phytochem.2005.05.006

Soylemezoglu, G., Demir, K., Inal, A., Gunes, A. (2009): Effect of silicon on antioxidant and stomatal response of two grapevine (Vitis vinifera L.) rootstocks grown in boron toxic, saline and boron toxic-saline soil. Scientia horticulturae. 123(2): 240-246. https://doi.org/10.1016/j.scienta.2009.09.005

Sun, H., Duan, Y., Qi, X., Zhang, L., Huo, H., Gong, H. (2018). Isolation and functional characterization of CsLsi2, a cucumber silicon efflux transporter gene. Annals of Botany. 122: 641-648. https://doi.org/10.1093/aob/mcy103

Sun, H., Guo, J., Duan, Y., Zhang, T., Huo, H., Gong, H. (2017): Isolation and functional characterization of CsLsi1, a silicon transporter gene in Cucumis sativus. Physiologia Plantarum. 159: 201-214. https://doi.org/10.1111/ppl.12515
Takahashi, E., Ma, J. F., Miyake, Y. (1990): The possibility of silicon as an essential element for higher plants. Comments on Agricultural Food Chemistry. 2: 99-102.

Torabi, F., Majd, A., Enteshari, S. (2015): The effect of silicon on alleviation of salt stress in borage (Borago officinalis L.). Soil Science and Plant Nutrition. 61: 788-798. https://doi.org/10.1080/00380768.2015.1005540

Toresano-Sánchez, F., Valverde-García, A., CamachoFerre, F. (2012): Effect of the Application of Silicon Hydroxide on Yield and Quality of Cherry Tomato. Journal of plant nutrition. 35(4): 567-590. https://doi.org/10.1080/ 01904167.2012.644375

Vercelli, M., Minuto, A., Minuto, G., Contartese, V., Devecchi, M., Larcher, F. (2017): The effects of innovative silicon applications on growth and powdery mildew control in soilless-grown cucumber (Cucumis sativus L.) and zucchini (Cucurbita pepo L.). Acta Physiologiae Plantarum. 39(6): 129. https://doi.org/10.1007/s11738-017-2426-5

Wagner, F. (1940): The importance of silicic acid for the growth of some cultivated plants, their metabolism, and their susceptibility to true mildews. Phytopathologische Zeitschrift.12(5).

Wang, H. S., Yu, C., Fan, P. P., Bao, B. F., Li, T., Zhu, Z. J. (2014): Identification of Two Cucumber Putative Silicon Transporter Genes in Cucumis sativus. Journal of Plant Growth Regulation. 34. https://doi.org/10.1007/s00344-014-9466-5

Wang, S., Liu, P., Chen, D., Yin, L., Li, H., Deng, X. (2015): Silicon enhanced salt tolerance by improving the root water uptake and decreasing the ion toxicity in cucumber. Frontiers in Plant Science. 6. https://doi.org/10.3389/fpls.2015.00759

Wang, Y., Wang, Y. (2018): Phytophthora sojae effectors orchestrate warfare with host immunity. Current Opinion in Microbiology, 46:7-13. https://doi.org/10.1016/j.mib.2018. 01.008

Ye, J., Yan, C., Liu, J., Lu, H., Liu, T., Song, Z. (2012): Effects of silicon on the distribution of cadmium compartmentation in root tips of Kandelia obovata (S., L.) Yong. Environmental Pollution. 162: 369-373. https://doi.org/10.1016/j.envpol.2011.12.002

Yin, L., Shiwen, W., Li, J., Tanaka, K., Oka, M. (2013): Application of silicon improves salt tolerance through ameliorating osmotic and ionic stresses in the seedling of Sorghum bicolor. Acta Physiologiae Plantarum. 35. https://doi.org/10.1007/s11738-013-1343-5

Zhang, C., Wang, L., Zhang, W., Zhang, F. (2013): Do lignification and silicification of the cell wall precede silicon deposition in the silica cell of the rice (Oryza sativa L.) leaf epidermis? Plant and Soil. 372. https://doi.org/10.1007/s11104013-1723-z

Zhu, Y., Gong, H. (2014): Beneficial effects of silicon on salt and drought tolerance in plants. Agronomy for Sustainable Development. 34, 455-472. https://doi.org/10.1007/s13593013-0194-1

Zuccarini, P. (2008): Effects of silicon on photosynthesis, water relations and nutrient uptake of Phaseolus vulgaris under $\mathrm{NaCl}$ stress. Biologia Plantarum. 521(1): 157-160. https://doi.org/10.1007/s10535-008-0034-3 